\title{
Uso dos recursos vegetais por agricultores familiares de Manacapuru (AM)
}

\begin{abstract}
Joanne R. COSTA ${ }^{1}$, Danielle MITJA ${ }^{2}$
RESUMO

Neste estudo é relatado o uso de espécies vegetais por onze famílias que vivem na área rural do município de Manacapuru, no Amazonas. Foi feita uma abordagem participativa durante as entrevistas, utilizando um formulário formal, que considerava toda a propriedade agrícola. No total, foram registradas 173 espécies, cuja maioria (101) apresentou uso medicinal. Sessenta e oito espécies são usadas na alimentação humana e vinte e duas espécies são utilizadas para construção de casas. Noventa e sete espécies $(56,1 \%)$ provêm somente dos pomares caseiros, 22 espécies $(12,7 \%)$ existem unicamente na floresta primária e 18 espécies $(10,4 \%)$ são provenientes das capoeiras. Algumas espécies são encontradas em diferentes ambientes. De fato, a vegetação da regiáo é capaz de prover diferentes recursos para os agricultores, os quais procuram alternativas que possam melhorar sua qualidade de vida. O plantio de espécies frutíferas é bastante comum e representa uma diversificaçáo e melhoria na qualidade alimentar. Porém, há necessidade de incentivo para o plantio de espécies florestais nativas. $\mathrm{O}$ aprendizado sobre as plantas medicinais é passado de geração a geração, sempre pelas mulheres.
\end{abstract}

PALAVRAS-CHAVE: Plantas medicinais, pomares caseiros, utilidade das plantas, sistemas agroflorestais.

\section{Use of vegetal resources by Family Farmers in Manacapuru, Amazonas state}

\section{ABSTRACT}

This study deals with the use of vegetal species by small farmers in the municipal district of Manacapuru, in the State of Amazonas.

We used a formal questionnaire for participative interviews which took into consideration the entire agricultural property.

Of the one hundred and seventy three species that we registered, the majority (101) presented medicinal use. Sixty-eight species are used for human nutrition and twenty-two for house building. Ninety-seven species $(56.1 \%)$ come exclusively from domestic orchards; twenty-two (12.7\%) exist only in the primary forest; and eighteen species (10.4\%) originate from fallows. Some species can be found in different environments.

Although the vegetation of the region under study is capable of providing farmers with necessary resources, their look for alternatives that might improve the quality of their lives. Planting fruit species is quite common and means both diversification and better food quality. It is however necessary to encourage planting native forest species. Knowledge and use of medicinal plants is always passed on from generation to generation by women.

KEYWORDS: Medicinal plants, home garden, usefulness of plants, agroforestry systems.

\footnotetext{
1 Embrapa Amazônia Ocidental, joanne.regis@cpaa.embrapa.br, Fone: (92) 3303-7909 - Fax: 3303-7820,End.: Rodovia AM-10, Km 29 Caixa Postal 319 - Manaus/AM- Brasil - 69010-970

2 Embrapa Cerrados, mitja@cpac.embrapa.br, Fone: (61) 3388-9898 - Fax: (92) 3388-9879. Rodovia BR 020 Km 18. Planaltina, DF - Brasil - CEP 73310-970, Caixa Postal: 08223
} 


\section{INTRODUÇÃO}

$\mathrm{Na}$ Amazônia, existe uma elevada quantidade de árvores, arbustos, ervas e cipós que contribuem, em vários aspectos, para o modo de vida das populaçôes (Le Cointe, 1947, Berg \& Silva, 1988, Rios et al., 2001). A diversidade biológica como fonte permanente de recursos constitui um imenso potencial para o desenvolvimento sustentável desta região. Pesquisadores têm demonstrado como a população tradicional que detém um amplo conhecimento sobre os recursos naturais, pode indicar os usos de espécies vegetais potenciais e ensinar novas opções para uso e manejo dos mesmos (Posey, 1992).

A Etnobotânica inclui todos os estudos concernentes à relação mútua entre populaçôes tradicionais e as plantas (Cotton, 1996) e apresenta como característica básica de estudo o contato direto com as populaçóes tradicionais, procurando uma aproximação e vivência que permitam conquistar a confiança das mesmas, resgatando, assim, todo conhecimento possível sobre a relação de afinidade entre o homem e as plantas de uma comunidade. A investigação etnobotânica pode desempenhar funçôes de grande importância como reunir informaçôes acerca de todos os possíveis usos de plantas, como uma contribuição para o desenvolvimento de novas formas de exploraçáo dos ecossistemas que se oponham às formas destrutivas vigentes (Schardong \& Cervi, 2000).

Portanto, o estudo etnobotânico é o primeiro passo para um trabalho multidisciplinar, a fim de se estabelecer quais são as espécies vegetais promissoras para pesquisas agropecuárias e florestais, justificando assim seu uso e sua conservação (Rodrigues \& Carvalho, 2001).

Considerando o conhecimento e a experiência no uso dos recursos vegetais de agricultores familiares amazonenses, este trabalho apresenta os resultados de um estudo sobre plantas e suas utilidades, realizado na zona rural do município de Manacapuru (AM).

\section{MATERIAL E MÉTODOS}

\section{ÁREA DE ESTUDO}

A área de estudo encontra-se entre o Rio Solimóes e a Rodovia AM-070 (Manaus - Manacapuru), Estado do Amazonas. Cinco agricultores entrevistados vivem no Ramal Nova Esperança, que liga o quilômetro 64 da Rodovia AM 070 ao lago do Calado, o qual desemboca no rio Solimóes. Os outros seis agricultores vivem no Ramal do Laranjal, que liga o quilômetro 62 da Rodovia AM-070 ao Rio Solimôes.

Segundo a classificação de Köppen, a área integra o quadro macroclimático do tipo $\mathrm{AmW}$, caracterizado por apresentar uma alta umidade (75\% a $86 \%)$, embora exista uma estação seca de pequena duração. As temperaturas médias mensais variam de 30 a $40{ }^{\circ} \mathrm{C}$. As chuvas ocorrem com grande intensidade (novembro a maio, estação chuvosa) e decrescem de intensidade (junho a outubro, estação seca) (RADAMBRASIL, 1978).

Silva et al. (1970) elaboraram um mapa do solo de grande parte dos municípios de Manacapuru e Iranduba. Baseados neste estudo, encontram-se nos ramais do Laranjal e Nova Esperança, os seguintes tipos de solos: Latossolos Amarelo textura pesada, fase floresta equatorial úmida, relevo suave ondulado e ondulado; Latossolo Amarelo, textura muito pesada, fase florestal equatorial úmida, relevo suave ondulado e ondulado; Podzol Hidromórfico; Latossolo Amarelo textura média, fase floresta equatorial úmida, relevo plano e suave ondulado.

A vegetação nativa da área pode ser classificada como floresta densa de terra firme caracterizada pela grande diversificaçâo de indivíduos arbóreos bem copados, fustes retos e portes elevados. Sua fisionomia é distingüida pelas gigantescas árvores que emergem do dossel superior (RADAMBRASIL, 1978).

\section{CARACTERIZAÇÃO AGROBOTÂNICA}

A caracterização agrobotânica das espécies úteis apresenta as seguintes informaçôes: nome vulgar, uso popular, partes usadas e ambientes em que são encontradas, como floresta, capoeiras, roças de mandioca, sistemas agroflorestais e pomares caseiros (quintais). Apesar dos pomares caseiros serem tipos de sistemas agroflorestais, neste trabalho eles foram separados daqueles sistemas implantados pelo Núcleo Agroflorestal do Instituto Nacional de Pesquisas da Amazônia (INPA), atuante na área desde 1992.

Foram consideradas apenas as plantas realmente usadas pelas famílias, não tendo sido incluídas aquelas com uso conhecido, mas não exploradas.

Os entrevistados foram indicados pela liderança comunitária por deterem maiores conhecimentos sobre plantas. Os indicados também sugeriram nomes de outras pessoas.

Foi utilizada uma abordagem participativa para a coleta das informaçôes, com base em um formulário. As entrevistas foram feitas percorrendo as propriedades agrícolas junto com os agricultores. No momento das entrevistas foram consideradas as informaçōes dos agricultores e de suas esposas. Após as entrevistas, foram feitas coletas das plantas, na companhia dos agricultores e preparadas exsicatas para posterior identificação no herbário do INPA.

\section{RESULTADOS E DISCUSSÃO}

\section{ESPÉCIES ENCONTRADAS}

Os resultados das entrevistas envolvendo 11 famílias de agricultores indicaram uma característica comum entre elas: a 
utilização de uma grande diversidade de espécies. Foi registrado o uso de 173 espécies (Tabela 1), nas 11 propriedades agrícolas. Em um estudo sobre populaçóes indígenas da comunidade de Novo Paraíso, localizada na Ilha de Bom Intento, no município de Benjamin Constant, Estado do Amazonas, Noda (2000) encontrou uma quantidade similar de espécies úteis (158) ao entrevistar 12 famílias.
Das 173 espécies encontradas neste estudo, 92 (52,6\%) são nativas da região e $80(45,08 \%)$ são introduzidas. Dezenove espécies são ruderais, sendo quatro introduzidas e 15 nativas. As ruderais se desenvolvem ao redor das casas e áreas cultivadas.

Tabela 1 - Espécies vegetais usadas por agricultores dos ramais Nova Esperança e Laranjal (Manacapuru,AM), em 2006. Origem: Introduzida ou Nativa. Hábito: Lenhosa; H: Herbácea; CH: Cipó Herbáceo, CL: Cipó Lenhoso (liana).

\begin{tabular}{|c|c|c|c|c|}
\hline Família & Nome científico & Nome vulgar & Origem & Hábito \\
\hline Lauraceae & Persea americana Mill. & Abacate & Introduzida & L \\
\hline Bromeliaceae & Ananas sativus $\mathrm{L}$. & Abacaxi & Introduzida & $\mathrm{H}$ \\
\hline Sapotaceae & Pouteria caimito (Ruiz e Pavon) Radlk & Abiu & Nativa & $\mathrm{L}$ \\
\hline Sapotaceae & Pouteria sp. & Abiurana & Nativa & L \\
\hline Clusiaceae & Mammea americana Linn & Abricó & Introduzida & L \\
\hline Olacaceae & Minquartia guianensis Aubl. & Acariquara & Nativa & L \\
\hline Arecaceae & Euterpe oleracea Mart. & Açaí-do-pará & Nativa & L \\
\hline Arecaceae & Euterpe precatoria Mart. & Açaí-do-amazonas & Nativa & L \\
\hline Malpighiaceae & Malpighia emarginata L. & Acerola & Introduzida & L \\
\hline Lamiaceae & Ocimum micranthum & Alfavaca & Introduzida & H \\
\hline Zingiberaceae & Curcuma longa $\mathrm{L}$. & Açafroa & Introduzida & $\mathrm{H}$ \\
\hline Malvaceae & Gossipidium herbaceum L. & Algodão roxo & Introduzida & H \\
\hline Portulacaceae & Portulaca pilosa L. & Amor crescido & Introduzida & $\mathrm{H}$ \\
\hline Myrtaceae & Eugenia stipitata Mc Vaugh & Araçá Boi & Nativa & L \\
\hline Vochysiaceae & Erisma sp. & Arara tucupi & Nativa & L \\
\hline Annonaceae & Annona coriacea Mart. & Araticum & Introduzida & L \\
\hline Meliaceae & Carapa guianensis Aubl. & Andiroba & Nativa & $\mathrm{L}$ \\
\hline Caesalpiniaceae & Dinizia excelsa Ducke. & Angelim pedra & Nativa & L \\
\hline Mimosaceae & Zygia racemosa (Ducke) Barneb.Grimes & Angelim rajado & Nativa & L \\
\hline Rutaceae & Ruta graveolens L. & Arruda & Introduzida & H \\
\hline Myrtaceae & Syzygium jambolana DC. & Azeitona & Introduzida & L \\
\hline Liliaceae & Aloe vera $\mathrm{L}$. & Babosa & Introduzida & H \\
\hline Clusiaceae & Rheedia sp. & Bacuri & Nativa & L \\
\hline Arecaceae & Oenocarpus bacaba Mart. & Bacaba & Nativa & L \\
\hline Arecaceae & Oenocarpus mapora Karsten & Bacabinha & Nativa & L \\
\hline Musaceae & Musa sp. & Banana & Introduzida & H \\
\hline Convolvulaceae & Ipomoea batatas Lam. & Batata doce & Introduzida & $\mathrm{CH}$ \\
\hline Annonaceae & Rollinia mucosa (Jacq.) Baill. & Biribá & Nativa & L \\
\hline Burseraceae & Protium crassipetalum Cuatr. & Breu branco & Nativa & L \\
\hline Asteraceae & Vernonia condensata Baker. & Boldo & Introduzida & L \\
\hline Lamiaceae & N.I. & Bolda & & $\mathrm{H}$ \\
\hline Arecaceae & Mauritia flexuosa L. & Buriti & Nativa & L \\
\hline Malpighiaceae & Banisteriopsis caapi (Spruce) Mort. & Cabi & Nativa & $\mathrm{CL}$ \\
\hline Sterculiaceae & Theobroma cacao L. & Cacau & Nativa. & L \\
\hline Piperaceae & Pothomorphe peltata (L.) Miq. & Capeba & Nativa* & $\mathrm{L}$ \\
\hline Poaceae & Cymbopogon citratus (DC) Stapf) & Capim-santo & Introduzida & H \\
\hline Apocynaceae & Aspidosperma album Vahl. & Carapanaúba & Nativa & L \\
\hline
\end{tabular}




\begin{tabular}{|c|c|c|c|c|}
\hline Família & Nome científico & Nome vulgar & Origem & Hábito \\
\hline Oxalidaceae & Averrhoa carambola L. & Carambola & Introduzida & $L$ \\
\hline Rubiaceae & Coffea arabica L. & Café & Introduzida & L \\
\hline Anacardiaceae & Anacardium occidentale L. & Caju & Nativa & L \\
\hline Anacardiaceae & Anacardium spruceanum Bth. ex. Engl. & Cajuí & Nativa & L \\
\hline Monimiaceae & Siparuna guianensis Aubl. & Capitiu & Nativa & L \\
\hline Portulacaceae & Talinum triangulare (Jacq.) Will. & Cariru & Introduzida* & $\mathrm{H}$ \\
\hline Lauraceae & Cinnamomum zeylanicum & Canela & Introduzida & $L$ \\
\hline Celastraceae & Goupia glabra Aubl. & Cupiúba & Nativa & L \\
\hline Lecythidaceae & Bertholletia excelsa H.B.K. & Castanha & Nativa & L \\
\hline Lecythidaceae & Lecythis pisonis Mori & Castanha Sapucaia & Nativa & L \\
\hline Lecythidaceae & Lecythis poiteaui Berg. & Castanha Jarana amarela & Nativa & L \\
\hline Apiaceae & Eryngium foetidum Linn. & Chicória & Introduzida* & $\mathrm{H}$ \\
\hline Bombacaceae & Scleronema micranthum Ducke & Cedrinho, Cardeiro & Nativa & $L$ \\
\hline Lamiaceae & Leonurus sibiricus L. & Cibalena & Introduzida & $\mathrm{H}$ \\
\hline Verbenaceae & Lippia alba (Mill) N.E.Br. & Cidreira & Nativa* & $\mathrm{H}$ \\
\hline Bignoniaceae & Adenocalymna alliaceum Miers. & Cipó Alho & Nativa & $\mathrm{CL}$ \\
\hline Convolvulaceae & Calycobolus ferrugineus (Choisy) House & Cipó Tuíra & Nativa & $\mathrm{CL}$ \\
\hline Arecaceae & Cocos nucifera L. & Coco & Introduzida & $\mathrm{L}$ \\
\hline Fabaceae & Copaifera reticulata Ducke & Copaíba & Nativa & $\mathrm{L}$ \\
\hline Crassulaceae & Kalanchoe brasiliensis Camb. & Corama & Introduzida & $\mathrm{H}$ \\
\hline Boraginaceae & Sympytum officinalis L. & Confrei & Introduzida & $\mathrm{H}$ \\
\hline Bignoniaceae & Arrabidaea chica Verlot. & Crajirú & Introduzida & $\mathrm{CL}$ \\
\hline Asteraceae & Tagetes erecta L. & Cravo de defunto & Introduzida & $\mathrm{H}$ \\
\hline Bignoniaceae & Crescentia cujete L. & Cueira & Nativa & L \\
\hline Amaranthaceae & Alternanthera tenella Coll. & Cuia mansa & Nativa* & H \\
\hline Fabaceae & Dipteryx odorata (Aubl.) Willd. & Cumaru & Nativa & $\mathrm{L}$ \\
\hline Acanthaceae & Justicia pectoralis Jacq. & Cumaruzinho & Introduzida & L \\
\hline Sterculiaceae & Theobroma grandiflorum (Spreng.) Schum. & Cupuaçu & Nativa & $\mathrm{L}$ \\
\hline Euphorbiaceae & Aparisthmium cordatum (Juss.) Baill. & Dema & Nativa & L \\
\hline Amaranthaceae & Pfaffia glomerata (Speag.) Peders. & Emenda osso & Nativa* & $\mathrm{H}$ \\
\hline Cecropiaceae & Cecropia sp. & Embaúba & Nativa & $L$ \\
\hline Piperaceae & Peperomia pellucida (L.) H.B.K. & Erva de Jabuti & Nativa* & H \\
\hline Loranthaceae & Struthanthus sp & Erva de passarinho & Introduzida & $\mathrm{H}$ \\
\hline Annonaceae & Bocageopsis multiflora (Mart.) R. E. Fries & Envira & Nativa & $\mathrm{L}$ \\
\hline Acanthaceae & Justicia scytophylla Leonard. & Eucalipto & Introduzida & $\mathrm{H}$ \\
\hline Moraceae & Artocarpus altilis (Park.) Fosberg. & Fruta-pão & Introduzida & $\mathrm{L}$ \\
\hline Caesalpiniaceae & Senna ocidentalis (L.) Link. & Fedegoso, Manjerioba & Nativa* & H \\
\hline Pedaliaceae & Sesamum indicum D.C. & Gergelim & Introduzida & $\mathrm{H}$ \\
\hline Annonaceae & Annona muricata Linn. & Graviola & Introduzida & $L$ \\
\hline Myrtaceae & Psidium guajava L. & Goiaba & Introduzida & $\mathrm{L}$ \\
\hline Melastomataceae & Bellucia grossularioides (L.) Triana. & Goiaba de Anta & Nativa & $L$ \\
\hline Sapindaceae & Paullinia cupana H.B.K. var. sorbilis Ducke & Guaraná & Nativa & $\mathrm{CL}$ \\
\hline Vochysiaceae & Erisma splendens Stafl. & Guaruba fofa & Nativa & $L$ \\
\hline Vochysiaceae & Qualea brevipedicellata Stafl. & Guaruba dura & Nativa & L \\
\hline Lamiaceae & Mentha cf. spicata L. & Hortelã pequeno & Introduzida & $\mathrm{H}$ \\
\hline
\end{tabular}




\begin{tabular}{|c|c|c|c|c|}
\hline Família & Nome científico & Nome vulgar & Origem & Hábito \\
\hline Scrophulariaceae & Bacopa sp. & Hortelãzinho & Nativa* & $\mathrm{H}$ \\
\hline Mimosaceae & Inga sp. & Ingá & Nativa & L \\
\hline Moraceae & Artocarpus heterophylla Lam. & Jaca & Introduzida & L \\
\hline Clusiaceae & Calophyllum brasiliensis Camb. & Jacareúba & Nativa & L \\
\hline Asteraceae & Eupatorium sp. & Japana roxa & Introduzida* & $\mathrm{H}$ \\
\hline Asteraceae & Eupatorium ayapana Vent. & Japana branca & Introduzida* & H \\
\hline Asteraceae & Spilanthes acmella L. & Jambu & Nativa* & $\mathrm{H}$ \\
\hline Myrtaceae & Eugenia sp. & Jambo & Introduzida & L \\
\hline Caesalpiniaceae & Hymenaea courbaril L. & Jatobá & Nativa & L \\
\hline Caesalpiniaceae & Caesalpinia ferrea Mart. & Jucá & Introduzida & L \\
\hline Solanaceae & Solanum sp. & Jurubeba & Nativa* $^{*}$ & L \\
\hline Rubiaceae & Genipa americana L. & Jenipapo & Introduzida & L \\
\hline Clusiaceae & Vismia japurensis Reich. & Lacre & Nativa & L \\
\hline Clusiaceae & Vismia cayennensis (Jacq.) Pers. & Lacre & Nativa & L \\
\hline Clusiaceae & Vismia cauliflora A.C. Smith. & Lacre & Nativa & L \\
\hline Clusiaceae & Vismia guianensis (Aubl.) Choisy & Lacre & Nativa & L \\
\hline Rutaceae & Citrus sinensis Persoon. & Laranja comum & Introduzida & L \\
\hline Rutaceae & Citrus sinenis Osbec. & Laranja paulista & Introduzida & L \\
\hline Rutaceae & Fortunela sp. & Laranja japonesa & Introduzida & L \\
\hline Rutaceae & Citrus sp. & Lima & Introduzida & L \\
\hline Rutaceae & Citrus limon (Christ.) Swingle & Limão comum & Introduzida & $\mathrm{L}$ \\
\hline Rutaceae & Citrus sp. & Limão tangerina & Introduzida & L \\
\hline Rubiaceae & Ferdinandusa paraensis Ducke & Louro Japurá & Nativa & L \\
\hline Rutaceae & Citrus aurantifolia (Christm.) Swingle. & Limão taiti & Introduzida & L \\
\hline Lauraceae & Licaria chrysophylla (Meissn) Kosterm. & Louro Cheiroso & Nativa & L \\
\hline Euphorbiaceae & Manihot sp. & Mandioca & Introduzida & L \\
\hline Fabaceae & Platymiscium duckei Huber & Macacaúba & Nativa & L \\
\hline Lamiaceae & Plectranthus amboinicus ( Lour.) Spreng. & Malvarisco, Hortelã Grande & Introduzida & H \\
\hline Caricaceae & Carica papaya L. & Mamão & Introduzida & L \\
\hline Cactaceae & Cereus cf. paraensis Hub. & Mandacaru & Introduzida & L \\
\hline Anacardiaceae & Mangifera indica L. & Manga & Introduzida & L \\
\hline Zingiberaceae & Zingiber officinale L. & Mangarataia & Introduzida & H \\
\hline Lamiaceae & Ocimum basilicum L. & Manjericão & Introduzida & $\mathrm{H}$ \\
\hline Araceae & Xanthosoma cf. auriculatum Regel. & Mão aberta & Introduzida & H \\
\hline Passifloraceae & Passiflora edulis Sims & Maracujá & Introduzida & $\mathrm{CL}$ \\
\hline Icacinaceae & Poraqueiba sericea Tul. & Mari & Nativa & L \\
\hline Simarubaceae & Simaruba amara Aubl. & Marupá & Nativa & L \\
\hline Cucurbitaceae & Cucumis anguria $\mathrm{L}$. & Maxixe & Introduzida & $\mathrm{CH}$ \\
\hline Chenopodiaceae & Chenopodium ambrosioides L. & Mastruz & Introduzida & $\mathrm{H}$ \\
\hline Violaceae & Paypayrola grandiflora Tul. & Matá-matá & Nativa & $\mathrm{L}$ \\
\hline Caesalpiniaceae & Cassia reticulata Willd. & Mata pasto & Nativa* & L \\
\hline Meliaceae & Swietenia macrophylla King & Mogno & Nativa & L \\
\hline Phytolacaceae & Petiveria alliacea L. & Mucuracaá & Nativa & $\mathrm{H}$ \\
\hline Iridaceae & Eleutherine bulbosa (Mill.) Urban & Murupaí & Nativa & H \\
\hline Malpighiaceae & Byrsonima chrysophylla Mart. & Murici & Nativa & L \\
\hline
\end{tabular}


Tabela 1 - Continuação

\begin{tabular}{|c|c|c|c|c|}
\hline Família & Nome científico & Nome vulgar & Origem & Hábito \\
\hline Piperaceae & Piper callosum Ruiz et Pav. & Óleo elétrico & Nativa & $\mathrm{H}$ \\
\hline Lamiaceae & Pogostemon patchouly Pellet. & Oriza & Introduzida & $\mathrm{H}$ \\
\hline Euphorbiaceae & Croton matourensis Aubl. & Orelha de burro & Nativa & L \\
\hline Bignoniaceae & Tabebuia serratifolia (Vahl.) Nichols. & Pau d'arco & Nativa & L \\
\hline Caesalpiniaceae & Bauhinia forficata L. & Pata de vaca & Introduzida & L \\
\hline Poaceae & Vetiveria zizanioides (L.) Nash) & Patchuli & Introduzida & $\mathrm{H}$ \\
\hline Amaranthaceae & Gomphrena globosa L. & Perpétua & Introduzida & $\mathrm{H}$ \\
\hline Apocynaceae & Ambelania acida Aubl. & Pepino do mato & Nativa & $\mathrm{L}$ \\
\hline Piperaceae & Piper nigrum L. & Pimenta -do-Reino & Introduzida & $\mathrm{CL}$ \\
\hline Caryocaraceae & Caryocar villosum (Aubl.) Pers. & Piquiá & Nativa & L \\
\hline Caryocaraceae & Caryocar glabrum (Aubl.) Pers. & Piquiarana & Nativa & L \\
\hline Euphorbiaceae & Jatropha curcas L. & Pinhão branco & Introduzida & L \\
\hline Euphorbiaceae & Jatropha podagrica Hook. & Pinhão pajé & Introduzida & L \\
\hline Euphorbiaceae & Jatropha gossypifolia L. & Pinhão roxo & Introduzida & L \\
\hline Myrtaceae & Eugenia uniflora $\mathrm{L}$. & Pitanga & Introduzida & L \\
\hline Sapindaceae & Talisia esculenta Radlk. & Pitomba & Nativa & $\mathrm{L}$ \\
\hline Costaceae & Costus spicatus (Jacq.) Sw. & Pobre velho & Introduzida & $\mathrm{H}$ \\
\hline Arecaceae & Bactris gasipaes H.B.K. & Pupunha & Nativa & $\mathrm{L}$ \\
\hline Rubiaceae & Borojoa sorbilis (Ducke) Cuatr & Puruí-Grande & Nativa & $\mathrm{L}$ \\
\hline Euphorbiaceae & Phyllanthus minutulus Muell. Arg. & Quebra pedra & Nativa* & $\mathrm{H}$ \\
\hline Apocynaceae & Geissospermum laeve Baill & Quina-quina & Nativa & L \\
\hline Verbenaceae & Stachytarpheta cayennensis (Rich.) Vahl. & Rinchão & Nativa* & $\mathrm{H}$ \\
\hline Lecythidaceae & Eschweilera coriacea (A. DC.) Mori & Ripeiro & Nativa & $\mathrm{L}$ \\
\hline Euphorbiaceae & Croton cajuçara Benth. & Sacaca & Introduzida & $\mathrm{L}$ \\
\hline Verbenaceae & Lippia grandis Schau. & Salve marajó & Introduzida & $\mathrm{L}$ \\
\hline Sapotaceae & Manilkara zapota (L.) P. van Royen & Sapotilha & Introduzida & L \\
\hline Fabaceae & Tephrosia adunca Benth & Sena & Nativa* & $\mathrm{H}$ \\
\hline Euphorbiaceae & Hevea brasiliensis Muell. Arg. & Seringa & Nativa & $\mathrm{L}$ \\
\hline Apocynaceae & Couma macrocarpa Barb. Rodr. & Sorva & Nativa & $\mathrm{L}$ \\
\hline Apocynaceae & Himathanthus sucuuba (Spruce) Woods & Sucuuba & Nativa & $\mathrm{L}$ \\
\hline Mimosaceae & Enterolobium schomburgkii (Benth) Benth. & Sucupira amarela & Nativa & $\mathrm{L}$ \\
\hline Combretaceae & Buchenavia sp. & Tanimbuca & Nativa & L \\
\hline Caesalpiniaceae & Tamarindus indica L. & Tamarina & Introduzida & L \\
\hline Anacardiaceae & Tapirira guianensis Aubl. & Tatapiririca & Nativa & $\mathrm{L}$ \\
\hline Anacardiaceae & Spondias mombin L. & Taperebá & Introduzida & L \\
\hline Rutaceae & Citrus nobilis Lour. & Tangerina & Introduzida & $\mathrm{L}$ \\
\hline Vochysiaceae & Qualea cyanea Ducke & Tintarana & Nativa & $\mathrm{L}$ \\
\hline Solanaceae & Lycopersicon esculentum Mill. & Tomate & Introduzida & $\mathrm{CH}$ \\
\hline Arecaceae & Astrocaryum tucuma Burret & Tucumã & Nativa & $\mathrm{L}$ \\
\hline Aristolochiaceae & Aristolochia sp. & Uecá & Introduzida & $\mathrm{CL}$ \\
\hline Humiriaceae & Endopleura uchi (Huber) Cuatr. & Uchi liso & Nativa & $\mathrm{L}$ \\
\hline Aristolochiaceae & Aristolochia triloba L. & Urubucaá & Introduzida & $\mathrm{CL}$ \\
\hline Bixaceae & Bixa orellana L. & Urucum & Introduzida & $\mathrm{L}$ \\
\hline Rubiaceae & Uncaria guianensis (Aubl.) Gmel. & Unha de gavião & Nativa & $\mathrm{CL}$ \\
\hline Scrophulariaceae & Scoparia dulcis L. & Vassourinha & Nativa* & $\mathrm{H}$ \\
\hline Zingiberaceae & Alpinia cf. nutans Posc. & Vindicá & Introduzida & $\mathrm{H}$ \\
\hline
\end{tabular}

* Espécies ruderais; N. I.: Não identificada. 
A maioria das espécies citadas é lenhosa (114-65,9\%), seguida das espécies herbáceas ( 46 espécies-26,6\%). Os cipós lenhosos (nove espécies-5,2\%) e os cipós herbáceos (quatro espécies-2,3\%) foram bem menos representados.

\section{USO DAS ESPÉCIES}

Do total das espécies registradas, 38,5\% apresentam uso múltiplo, tais como: azeitona (Syzygium jambolana), açaí (Euterpe oleraceae e Euterpe precatoria), goiaba (Psidium guajava), laranja (Citrus sinensis), manga (Mangifera indica) e pinhão branco (Jatropha curcas) que são medicinais, mas também servem para alimentação do homem e de animais. O piquiá (Caryocar villosum) serve para alimentação humana, fabricação de objetos, móveis e construçấo de casas. A goiaba de anta (Bellucia grossularioides) é utilizada para alimentação animal, fabricação de objetos e lenha. O cipó alho (Adenocalymna alliaceum) tem usos mágicos, medicinais e alimentícios.

Mais da metade das plantas citadas tem uso medicinal (101 espécies), o que representa 58,38\%. Há 55 espécies cujo uso é somente medicinal, correspondendo a $31,8 \%$ das espécies registradas. Em populaçóes indígenas da comunidade de Novo Paraíso (Amazonas) foi observado por Noda (2000), um número menor (71) de plantas medicinais. Percebe-se uma grande importância da natureza para a saúde e alimentação dos agricultores daquela área, que não contam com hospitais e médicos nas proximidades das suas residências. Quando há uma pessoa doente na família, os remédios naturais são os primeiros a serem usados. Somente no caso de agravamento do problema, os agricultores procuram os hospitais. Segundo os entrevistados, atualmente, o conhecimento sobre o valor medicinal das ervas é bem menor do que na época de seus avós, devido à erosão de modos e costumes tradicionais. A dificuldade de acesso aos medicamentos alopáticos tem contribuído bastante para a continuação do uso dos remédios naturais.

As mulheres mostraram-se como as maiores detentoras de conhecimento sobre plantas medicinais, sendo as responsáveis pela produção de mudas e preparação dos chás. Elas seguem algumas recomendaçôes importantes passadas por suas mães, a fim de evitar problemas de saúde, tais como: evitar o uso de plantas desconhecidas; nunca aumentar a dose de uma receita, pois há perigo de envenenamento e nunca usar internamente plantas recomendadas para uso externo e usar somente plantas saudáveis. A mulher é a responsável pela transmissão dos conhecimentos sobre as plantas medicinais e o faz para qualquer interessado, mas principalmente para as filhas.

Sessenta e oito espécies são usadas na alimentação da família, sendo a maioria delas composta pelas frutíferas. Apesar do número de espécies ser menor do que aquele encontrado na categoria alimento (91 espécies) por Noda (2000) em estudo na comunidade de Novo Paraíso (Amazonas), a quantidade registrada neste estudo é importante pois garante uma melhoria na qualidade alimentar, um aumento da renda familiar ou a entrada de outros produtos necessários, já que a produção pode ser vendida ou trocada nos próprios ramais ou na feira de Manacapuru.

Os agricultores costumam criar gado, patos, galinhas, marrecos e estes animais também são beneficiados pela existência de um bom número de espécies vegetais fornecedoras de alimento. Dezessete espécies são utilizadas para alimentar os animais. Apenas sete espécies são usadas para construção de móveis. Árvores como angelim pedra (Dinizia excelsa) e cedrinho (Scleronema micranthum), segundo os agricultores, apresentam a qualidade necessária para se ter um bom produto, mas são difíceis de serem encontradas por já terem sido bastante exploradas.

Onze espécies citadas são utilizadas para fabricação de variados tipos de objetos, como caixas, brinquedos, cuias, colheres etc. Apenas quatro espécies são usadas para construção de cerca para delimitar terrenos. Quando resta material proveniente da construçáo de casas e móveis, utiliza-se esta sobra para substituir cercas já deterioradas.

Vinte e duas espécies são utilizadas para construção das casas. Para os produtores é mais econômico construir suas casas com madeira, pois o material pode ser encontrado nas proximidades. Outro fator citado é que no clima quente da regiáo uma casa de madeira é mais ventilada.

Foi verificado um maior conhecimento dos homens sobre as espécies madeiráveis. Os agricultores mencionaram que tem havido uma diminuiçấo qualitativa e quantitativa das plantas madeiráveis, devido à exploração intensa nas florestas próximas às suas casas. Esta exploração não é feita somente pelos moradores do local, mas por pessoas que vêm de fora, contratados por madeireiras. O desaparecimento destas espécies provém também dos desmatamentos feitos pelos próprios agricultores para a implantação de cultivos anuais, como a mandioca (Manihot esculenta Crantz).

Doze espécies, provenientes da floresta e dos pomares caseiros e usadas com maior freqüência no passado, estão sendo menos procuradas com o passar do tempo. Atualmente as mais usadas são para casos de "mau-olhado" em crianças muito pequenas.

A lenha proveniente das capoeiras é usada, principalmente, na ocasiâo da preparação da mandioca. Quinze espécies são utilizadas para fazer fogo. Rios (2001), em um estudo realizado na comunidade de Benjamin Constant, no Estado do Pará, classificou as plantas encontradas em capoeiras em 11 categorias de uso, destacando-se o emprego para combustível, com 84 espécies que servem para lenha, valor bem superior ao encontrado em Manacapuru. 
A valorizaçáo dos recursos vegetais pelas comunidades é resultado de representaçôes feitas sobre a importância da floresta e do contexto em que tais representaçóes são construídas (Godelier, 1984). Segundo Luckert e Campbell (2002) citados por Medina (2004) "os valores que as pessoas atribuem para os recursos naturais são chaves para compreender a racionalidade de suas decisóes". É neste sentido que muitos moradores da floresta podem estar prontos para convertê-la em diferentes usos, se a opção está dentro de seu alcance e se as circunstâncias os levam a isto (Henkemans, 2001).

\section{A PARTE USADA DAS PLANTAS}

Das 173 espécies registradas, as folhas são utilizadas em 67 espécies e os frutos em 66 espécies. De 51 espécies são retiradas partes do caule para aproveitamento. As cascas de 25 espécies, as raízes de 21 espécies e os galhos de 17 espécies são usados em remédios caseiros. As sementes de 16 espécies e as flores de apenas nove espécies têm alguma utilidade para os agricultores.

Muitas vezes, são usadas diferentes partes de uma mesma planta. Somente três espécies são inteiramente aproveitadas: cuia mansa (Alternanthera tenella), erva de jabuti (Peperomia pellucida) e sena (Tephrosia adunca).

\section{PROCEDÊNCIA DAS PLANTAS}

O extrativismo é praticado, mas é uma atividade secundária. Em sua maioria, as plantas são cultivadas nos pomares caseiros ou quintais agroflorestais (119 espécies) e nos sistemas agroflorestais (22 espécies) implantados por pesquisadores do INPA. Em um estudo desenvolvido em Boa Vista do Ramos (AM) sobre o uso de remédios caseiros, Midei e Mello (dados não publicados) verificaram que mais da metade da populaçáo amostrada (53\%) utiliza as plantas dos quintais e da mata primária, sendo que as cultivadas nos quintais são mais freqüentemente utilizadas (61\%). Isto demonstra o interesse dos agricultores em plantar, pois segundo eles, significa uma melhoria na alimentação e uma entrada de recursos financeiros importantes para uma melhor qualidade de vida. Os pomares caseiros, também chamados pelos agricutores de "sítio", são formas tradicionais de sistemas agroflorestais, localizados junto às residências. Apresentam uma baixa densidade de indivíduos por espécie e uma alta quantidade de espécies, principalmente de frutíferas, que formam diferentes estratos. A disposição das plantas é irregular no terreno. Os produtos provenientes dos pomares são usados, em sua maioria, para consumo familiar, sendo os excedentes vendidos. As mudas e sementes são conseguidas com os vizinhos, amigos e poucas vezes foram compradas. Para Guillaumet et al. (1990), que estudaram três pomares caseiros no Amazonas, encontrando 61 espécies diferentes, o sítio é um sistema complexo e bem adaptado às condiçôes do ambiente natural. Há um constante aproveitamento da diversidade tanto específica como estrutural, levando a uma ocupação máxima do espaço e a utilização ótima do ciclo sazonal.

Trinta e cinco espécies são coletadas na floresta primária, a qual fornece madeira para construçáo de benfeitorias a serem usadas na propriedade ou para comercialização. Apesar dos agricultores mencionarem a ausência das espécies madeiráveis nas áreas onde antigamente havia uma alta quantidade, não se percebeu interesse em seu plantio, o que está relacionado à demora do retorno econômico.

Durante o período de descanso da terra usada para cultivos agrícolas, quando se formam as capoeiras, áreas de regeneração natural, 24 espécies são aproveitadas para diferentes fins pelos agricultores entrevistados. Assim, considera-se que a área não foi abandonada completamente, sendo um habitat importante de plantas úteis. Em um estudo realizado por Rios et al. (2001), nas capoeiras da comunidade de Benjamin Constant (Pará), foram registradas cerca de 200 espécies de plantas úteis reconhecidas pelos agricultores familiares, muitas delas apresentando usos múltiplos. Vieira et al. (1996) identificaram 150 espécies úteis em capoeiras no município de Peixe-Boi (Pará). Ali os colonos praticam a coleta de plantas medicinais, o corte de árvores para lenha e carvão e mesmo a caça de animais, até que a capoeira seja removida para dar lugar a novos cultivos.

Em um levantamento feito em capoeiras de antigas roças dos índios Kayapó foram encontradas 368 espécies, das quais 94\% destinavam-se ao uso medicinal (Posey 1986), o que mostra a importância das capoeiras para aquela etnia. Segundo Finegan (1992), o manejo de capoeiras é viável, entretanto, nem todos os locais têm a mesma potencialidade. No caso de Manacapuru, a capoeira fornece algum tipo de rendimento como a produçáo de frutos de certas espécies como bacaba (Oenocarpus bacaba), tucumã (Astrocaryum tucuma), uchi liso (Endopleura uchi) e como área que atrai caça (cotia, paca, tatu e veado). Outros usos citados na literatura (Rios et al., 2001), como produção de madeira para construção de casas, barracas, currais, chiqueiros; produção de cipós e fibras para confecçấo de paneiros e peneiras náo foram mencionados pelos agricultores de Manacapuru. Provavelmente, isso seja resultado do pouco conhecimento sobre algumas espécies ou de recursos para sua exploração.

Nas roças de mandioca estudadas, apenas quatro espécies foram citadas, incluindo a mandioca, que é usada, principalmente, para produção de farinha para subsistência ou venda. As outras três espécies, a castanha (Bertholletia excelsa), o piquiá (Caryocar villosum) e o tucumã (Astrocaryum tucuma) foram encontradas nas áreas no momento do corte e queima mas foram preservadas. Pereira e Lescure (1994) estudaram uma comunidade ribeirinha no Médio Solimôes (Amazonas) e, como neste trabalho, também observaram um tendência à super-especialização das roças de mandioca, provavelmente 
em função do caráter fortemente comercial desta produção. Segundo os autores, diferentemente do sistema indígena original, as roças estudadas eram constituídas basicamente de plantas de mandioca, apenas algumas plantas de outras culturas tradicionais, tais como, banana (Musa sp.), cará (Dioscorea sp.), abacaxi (Ananas sativus) e cana-de-açúcar (Saccharum officinarum), cultivadas pelas mulheres para o consumo da família.

Vinte e sete espécies tiveram procedência de dois ambientes diferentes, por exemplo: Cipó tuíra (Calycobolus ferrugineus), encontrado no pomar e na capoeira, matá-matá (Paypayrola grandiflora), cupiúba (Goupia glabra) e pau d'arco (Tabebuia serratifolia), encontrados na floresta e capoeira, andiroba (Carapa guianensis), encontrada no pomar e na floresta e bacuri (Rheedia sp.) que foi encontrada no pomar e nos sistemas agroflorestais. Apenas duas espécies apresentaram procedência de três ambientes: piquiá (mandioca, pomar caseiro, $\mathrm{SAFs}$ ) e castanha (floresta, roça de mandioca e SAFs).

\section{CONSERVAÇÃO DAS ESPÉCIES}

Para a maioria das plantas, seu uso não provoca a destruição do indivíduo que continua sendo uma fonte produtora de recursos para o futuro. Nos casos de uso do fruto ou de sementes é possível que a regeneração das espécies seja afetada.

No caso das espécies madeiráveis, que são totalmente derrubadas e cujos indivíduos novos apresentam um crescimento muito demorado, a exploração desordenada da espécie está empobrecendo as florestas próximas, sendo necessário ir cada vez mais longe para se extrair madeira.

Em geral, o uso dos recursos florestais é igualmente ligado ao desmatamento realizado para instalação dos cultivos agrícolas. O ambiente necessário para o crescimento da maioria das espécies madeiráveis é, portanto, perturbado. Por enquanto, mesmo estando consciente da diminuição de plantas madeiráveis em seus terrenos, os agricutores ainda náo têm interesse em seu plantio. Isso porque necessitam de cultivos que forneçam um retorno financeiro mais rápido para garantir sua sobrevivência.

Percebe-se que a vegetação existente oferece um apreciável potencial capaz de ser usado de diferentes maneiras, para os mais diferentes objetivos. A sobrevivência da população que vive próxima à floresta, geralmente de baixa renda, é garantida a partir de sua relação com a natureza.

\section{CONCLUSÕES}

Os agricultores entrevistados são detentores de um rico saber sobre os recursos vegetais, os quais representam um meio para criar melhores condiçóes de vida.
De fato, a vegetação da área é capaz de prover diferentes recursos para os agricultores. $\mathrm{O}$ uso de espécies medicinais é bastante comum e o plantio de sistemas agroflorestais tem representado a diversificação na produção, a oferta de alimentos ao longo do ano e a melhoria na qualidade alimentar das famílias.

$\mathrm{O}$ aprendizado sobre as plantas medicinais é passado de geração a geração e sempre pelas mulheres.

O uso das espécies encontradas nas capoeiras é importante. Sugere-se que sejam feitos estudos mais aprofundados sobre as espécies componentes das capoeiras e tecnologias com baixo custo para maior aproveitamento desse recurso, o que poderia fornecer mais benefícios para os agricultores.

Observou-se a necessidade de incentivo para o plantio de espécies florestais nativas.

\section{BIBLIOGRAFIA CITADA}

Berg, M.E.V.D.; Silva, M.H.L. 1988. Contribuição ao conhecimento da flora medicinal de Roraima. Acta Amazonica, 18: 23-35.

Cotton, C.M. 1996. Ethnobotany: principles and applications. J. Wiley New York. 320 pp.

Finegan, B. 1992. The management potential of neotropical secondary lowland rain forest. Forest Ecology and Management. 47: 295-321.

Godelier, M. 1984. L'Idéel et le Matériel. Fayard. Paris. 348 pp.

Guillaumet, J.L.; Grenand, P.; Bahri, S.; Grenand, F.; Lourd, M.; Santos, A.A. dos; Gely, Y A. 1990. Les jardins-vergers familiaux d'Amazonie Centrale: um exemple de l'utilisation de l'espace. Turrialba, 40: 63-81.

Henkemans, A.B. 2001. Tranquilidad and Hardship in the Forest: Livelihoods and Perceptions of Camba Forest Dwellers in the Northern Bolivian Amazon. PROMAB. Scientific Serie,s 5: $1-42$.

Le Cointe, P. 1947. Árvores e plantas úteis (Indigenas e aclimatadas). Brasiliana, Biblioteca Pedagógica Brasileira, Amazônia Brasiliense III, Editora Nacional, São Paulo, 506 pp.

Medina, G. 2004. Ocupação cabocla e extrativismo madeireiro no Alto Capim: uma estratégia de reproduçấo camponesa. Acta Amazonica, 34: 309 - 318.

Noda, S. N. 2000. Na terra como na água, organização e conservação de recursos naturais terrestres e aquáticos em uma comunidade da Amazônia brasileira. Tese de doutorado. Universidade Federal do Mato Grosso, Cuiabá, MT. 182 pp.

Pereira, H.; Lescure, J.P.1994. Extrativismo e agricultura: as escolhas de uma população Kokama do Médio Solimões. Revista Universidade do Amazonas Série: Ciências Agrárias, 1: 1 - 9.

Posey, D. A. 1986. Etnoentomologia de tribos indígenas da Amazônia. p. 251-272. In: Suma Etnológica Brasileira. Vozes/ Finep. Petrópolis.

Posey, D.A.1992.Traditional knowledge, conservation, and the rain forest harvest. 325pp. In: M. Plotkin \& L. Famolare 
(eds). Sustainable harvest and marketing of rain florest products. Washington, Island Press/Conservation International.

RADAMBRASIL, 1978. Folha SA 20 Manaus. Levantamento de recursos naturais. DNPM/RADAMBRASIL. Rio de Janeiro, RJ. 18:1-628.

Rios, M.; Martins-Da-Silva, R. C. V.; Sabogal, C.; Martins, J.; Silva, R. N.; Brito, R. R.; Brito, I. M. de; Brito, M. de F. C.; Silva, J. R.; Ribeiro, R. T. 2001. Beneficios das plantas da capoeira para a comunidade de Benjamin Constant, Pará, Amazônia brasileira. CIFOR. Belém, PA. 54 pp.

Rodrigues, V.E.G.; Carvalho, D.A. 2001. Levantamento etnobotânico de plantas medicinais no domínio do cerrado na região do Alto Rio Grande - Minas Gerais. Ciência e Agrotecnologia, 25: 102123.
Schardong, R.M.F.; Cervi, A.C. 2000. Estudos etnobotânicos das plantas de uso medicinal e místico na comunidade de São Benedito, Bairro São Francisco, Campo Grande, MS, Brasil. Acta Biológica Paranaense, 29: 187-217.

Silva, B.N.R.; Araújo, J.V.; Rodrigues,T.E.; Falesi, I.C.; Rego, R. S. 1970. Os solos da área Cacau-Pirêra-Manacapuru. Solos da Amazônia. IPEAN. 2 (3), Belém, 198 pp.

Vieira, I.C.G.; Salomão, R.P.; Nepstad, D.C.; Roma, J.; Rosa, N.1996. O renascimento da floresta no rastro da agricultura. Ciência Hoje, 119: 38-44.

Recebido em: 23/11/2007

Aceito em:07/10/2009 